\title{
Amiloride ameliorates muscle wasting in cancer cachexia through inhibiting tumor- derived exosome release
}

\author{
Lin Zhou', Tong Zhang ${ }^{1}$, Wei Shao ${ }^{2}$, Ruohan Lu', Lin Wang ${ }^{3}$, Haisheng Liu', Bin Jiang ${ }^{4}$, Shiqin Li ${ }^{5}$, Huiqin Zhuo ${ }^{6}$,
} Suheng Wang ${ }^{7}$, Qinxi Li ${ }^{4}$, Caihua Huang ${ }^{8^{*}}$ and Donghai Lin ${ }^{1,9^{*}}$ (D)

\begin{abstract}
Background: Cancer cachexia (CAC) reduces patient survival and quality of life. Developments of efficient therapeutic strategies are required for the CAC treatments. This long-term process could be shortened by the drugrepositioning approach which exploits old drugs approved for non-cachexia disease. Amiloride, a diuretic drug, is clinically used for treatments of hypertension and edema due to heart failure. Here, we explored the effects of the amiloride treatment for ameliorating muscle wasting in murine models of cancer cachexia.

Methods: The CT26 and LLC tumor cells were subcutaneously injected into mice to induce colon cancer cachexia and lung cancer cachexia, respectively. Amiloride was intraperitoneally injected daily once tumors were formed. Cachexia features of the CT26 model and the LLC model were separately characterized by phenotypic, histopathologic and biochemical analyses. Plasma exosomes and muscle atrophy-related proteins were quantitatively analyzed. Integrative NMR-based metabolomic and transcriptomic analyses were conducted to identify significantly altered metabolic pathways and distinctly changed metabolism-related biological processes in gastrocnemius.

Results: The CT26 and LLC cachexia models displayed prominent cachexia features including decreases in body weight, skeletal muscle, adipose tissue, and muscle strength. The amiloride treatment in tumor-bearing mice distinctly alleviated muscle atrophy and relieved cachexia-related features without affecting tumor growth. Both the CT26 and LLC cachexia mice showed increased plasma exosome densities which were largely derived from tumors. Significantly, the amiloride treatment inhibited tumor-derived exosome release, which did not obviously affect exosome secretion from non-neoplastic tissues or induce observable systemic toxicities in normal healthy mice. Integrative-omics revealed significant metabolic impairments in cachectic gastrocnemius, including promoted muscular catabolism, inhibited muscular protein synthesis, blocked glycolysis, and impeded ketone body oxidation. The amiloride treatment evidently improved the metabolic impairments in cachectic gastrocnemius.
\end{abstract}

\footnotetext{
* Correspondence: huangcaihua@xmut.edu.cn; dhlin@xmu.edu.cn

${ }^{8}$ Research and Communication Center of Exercise and Health, Xiamen

University of Technology, Xiamen 361024, China

'Key Laboratory for Chemical Biology of Fujian Province, MOE Key Laboratory

of Spectrochemical Analysis \& Instrumentation, College of Chemistry and

Chemical Engineering, Xiamen University, Xiamen 361005, China

Full list of author information is available at the end of the article
}

(c) The Author(s). 2021 Open Access This article is licensed under a Creative Commons Attribution 4.0 International License, which permits use, sharing, adaptation, distribution and reproduction in any medium or format, as long as you give appropriate credit to the original author(s) and the source, provide a link to the Creative Commons licence, and indicate if changes were made. The images or other third party material in this article are included in the article's Creative Commons licence, unless indicated otherwise in a credit line to the material. If material is not included in the article's Creative Commons licence and your intended use is not permitted by statutory regulation or exceeds the permitted use, you will need to obtain permission directly from the copyright holder. To view a copy of this licence, visit http://creativecommons.org/licenses/by/4.0/ The Creative Commons Public Domain Dedication waiver (http://creativecommons.org/publicdomain/zero/1.0/) applies to the data made available in this article, unless otherwise stated in a credit line to the data. 
Conclusions: Amiloride ameliorates cachectic muscle wasting and alleviates cancer cachexia progression through inhibiting tumor-derived exosome release. Our results are beneficial to understanding the underlying molecular mechanisms, shedding light on the potentials of amiloride in cachexia therapy.

Keywords: Amiloride, Cancer cachexia, Muscle wasting, Exosome, Exosome-release inhibition

\section{Introduction}

Cachexia is a systemic metabolic syndrome defined by involuntary body weight and skeletal muscle loss (with or without fat loss) and cannot be fully reversed by conventional nutritional supplementations [1]. Driven by a complicated combination of endocrine and metabolic disorders as well as central nervous system perturbations, cachexia is characterized by several predominant features including reduced food intake, decreased muscle mass, excess catabolism, unnecessary energy expenditure, and hyper-inflammatory response [2]. Pathologic mechanisms of cancer cachexia are closely related to activation of proteolysis, autophagy, lipolysis, and inflammation [3].

Cachexia is usually associated with chronic and malignant diseases, including kidney disease, heart failure, neurological disease, chronic obstructive pulmonary disease, AIDS, and, especially, cancer [4]. As one of the leading causes of death worldwide, cancer accounts for an estimated 9.6 million deaths in 2018, and nearly $80 \%$ of cancer patients are affected by cachexia in different grades [5]. Cancer cachexia (CAC) directly causes about one third of fatalities in cancer patients with significant skeletal muscle wasting [6]. Efficient therapeutic strategies for the CAC treatments are urgently needed [4]. Recently, mounting researches indicated that tumorderived exosomes contribute to cancer cachexia through mediating the cross-talk between tumors and distally located skeletal muscles, resulting in decreased muscle weight, impaired organismal function, suppressed therapeutical response, and reduced quality of life, as well as remarkably enhanced cancer-related mortality [7-10]. These works might provide a new strategy for the CAC treatments based on inhibition of tumor-derived exosomes release.

Amiloride is an old drug with potassium-sparing diuretic function (upon inhibition of $\mathrm{Na}^{+} / \mathrm{H}^{+}$and $\mathrm{Na}^{+} / \mathrm{K}^{+}$exchangers), which has been clinically employed in the treatments of hypertension, hypokalemia, edema, and congestive heart failure for decades [11]. Most importantly, amiloride can inhibit exosome release from cells and reverse exosome-promoted pathogenic processes, including autoimmune disease and immuno-suppressive regulation [12, 13]. However, no attempts have been reported to ameliorate cachectic muscle wasting through inhibiting tumor-derived exosome release. Given that tumor-derived exosomes are implicated in mediating cachectic muscle wasting, we speculated that amiloride might have some effects for ameliorating muscle wasting in cancer cachexia.

In the present work, we sought to determine whether amiloride was able to ameliorate muscle wasting in murine cachexia models. Furthermore, we addressed molecular mechanisms of tumor-derived exosomes promoting muscle wasting by integrative metabolomic and transcriptomic analyses, which provides the mechanistic rationale for exploiting clinical potentials of amiloride for improving the CAC treatments. Our results demonstrated that the amiloride treatment could significantly ameliorate muscle wasting in cancer cachexia and thus alleviate the CAC progression through inhibiting tumor-derived exosome release.

\section{Methods}

\section{Patient blood sample collection}

Patient blood samples were firstly kept in anticoagulation (100 mM sodium citrate) tubes and then centrifuged $\left(1000 \mathrm{~g}, 10 \mathrm{~min}, 4^{\circ} \mathrm{C}\right)$ to obtain platelet-free plasma within $2 \mathrm{~h}$ after blood collection. The plasma was aliquoted and collected in cryovials and kept at $-80{ }^{\circ} \mathrm{C}$ until used.

\section{Exosome isolation and characterization}

Exosomes in either patient/mouse plasma or culture media of CT26/LLC cells were isolated by ultracentrifugation $[14,15]$. A Beckman Coulter XE-90K Ultracentrifuge equipped with an SW 41 Ti rotor was used for the ultracentrifugation. Particle sizes of exosomes were analyzed using ZetaView ${ }^{\circ}$ Nanoparticle Tracking Analyzer (Particle Metrix GmbH, Meerbusch, Germany) and FEI Tecnai 20 transmission electron microscope (Thermo Fisher, USA) according to the manufacturer's manual [16] or the published protocol [17], respectively.

\section{Exosome density and purity measurements}

Exosome density and purity were analyzed with a twochannel high-sensitivity nano flow cytometer (HSFCM) according to the protocol described previously [15]. The HSFCM was developed by the Laboratory of Professor XM Yan from College of Chemistry and Chemical Engineering, Xiamen University, which has been commercialized by NanoFCM Inc, China. To assess exosome purities, 1\% final volume of Triton X-100 (Sigma-Aldrich, USA) was added to exosome suspensions, and the 
HSFCM measurement was repeated after incubation of $30 \mathrm{~min}$ on ice.

\section{Cell culture}

The LLC, CT26, and HEK 293T cells were purchased from the China Center for Typical Culture Collection (CCTCC). C2C12 cells were provided by Stem Cell Bank, Chinese Academy of Sciences. LLC and CT26 cells were cultured in DMEM and RPMI-1640, respectively. Both culture media were supplemented with 100 units/ $\mathrm{mL}$ penicillin, $100 \mu \mathrm{g} / \mathrm{mL}$ streptomycin, and $10 \%$ fetal bovine serum (Hyclone, USA). All cells were cultured in a humidified atmosphere of $5 \% \mathrm{CO}_{2}$ at $37^{\circ} \mathrm{C}$. Culture media of LLC and CT26 cells were collected and centrifuged $\left(1000 \mathrm{~g}, 5 \mathrm{~min}, 4^{\circ} \mathrm{C}\right)$ after $48 \mathrm{~h}$ of culture. $\mathrm{C} 2 \mathrm{C} 12$ myoblasts were cultured to $85-90 \%$ confluence in DMEM growth medium. Myoblast differentiation was induced by incubation for $96 \mathrm{~h}$ in DMEM supplemented with $2 \%$ heat-inactivated horse serum. C2C12 myoblasts were used within ten generations of culture.

\section{Lentiviral expression of shRNA in tumor cells}

The pLKO.1-puro lentivirus vector was used to express the shRNAs. The virus was generated by four cotransfected plasmids, including the lentiviral vector, pMDLg/pRRE, pRSV-Rev, and pMD2 in HEK 293T cells. At $48 \mathrm{~h}$, virus-containing supernatants were collected for transduction in CT26 and LLC cells. The shRNAs against mouse Rab27a and Rab27b were $5^{\prime}$ GCTTCTGTTCGACCTGACAAA-3' and 5'-GCTT CTGGACTTAATCATGAA-3', respectively.

\section{Animal experiments}

To assess the effects of amiloride for ameliorating muscle atrophy in vivo, we constructed murine models of CT26 (colon) and LLC (lung) cancer cachexia (Fig. S1). Adult (age 6-8 weeks) C57BL/6 and BALB/c male mice were purchased from Shanghai SLAC Laboratory Animal Co., Ltd. Mice were individually housed, acclimated to their cages and human handling for 1 week before animal experiments, and maintained in conditions of constant temperature and 12-h light/12-h dark cycles.

Tumor cells were subcutaneously injected into the right flank of mice on day 0 . In detail, BALB/c mice were inoculated with the CT26 cells $\left(1.0 \times 10^{6} / 100 \mu \mathrm{L}\right)$ to induce colon cancer cachexia, while $\mathrm{C} 57 \mathrm{BL} / 6$ mice were inoculated with the LLC cells $\left(7 \times 10^{5} / 100 \mu \mathrm{L}\right)$ to induce lung cancer cachexia. Both BALB/c and C57BL/6 mice showed palpable tumors (about $5 \mathrm{~mm}$ in diameter) on day 9 after the inoculation. Both CT26-bearing mice and LLC-bearing mice were randomly divided into 2 groups ( $n=8$ per group): one group of mice intraperitoneally injected daily with PBS from day 9 (CAC mice), another group of mice intraperitoneally injected daily with amiloride dissolved in PBS at a dose of $2 \mathrm{mg} / \mathrm{kg}$ (AM mice). Furthermore, The Rab27 knock-down tumor cells were subcutaneously injected into the mice following the same procedure (KD mice). Similarly, normal control mice were injected with PBS on day 0 (NOR mice). Both the KD and NOR mice were intraperitoneally injected with PBS daily from day 9.

Mouse body weights were monitored every 3-day posttumor implantation, and food intakes were measured every day. Tumor volumes were calculated every 3-day post-tumor implantation using the formula: tumor volume $\left(\mathrm{mm}^{3}\right)=0.52 \times$ length $\times$ width $^{2}$, in which the length and perpendicular width were measured with a vernier caliper. Forelimb grip forces were measured with a Grip Strength Meter (YLS-13A, Shandong Academy of Medical Sciences, China). For each mouse, the grip strength was defined as the average of five successive measurements. On day 30, the mice were sacrificed. Both tumors and gastrocnemius were removed, weighed, and quickly frozen in liquid nitrogen for subsequent analysis. The mouse blood samples were firstly kept in anti-coagulation (100 mM sodium citrate) tubes and pro-coagulation tubes and then centrifuged $(1000 \mathrm{~g}, 10$ min, $4{ }^{\circ} \mathrm{C}$ ) to obtain platelet-free plasma and serum, respectively, within $2 \mathrm{~h}$ after blood collection. The plasma exosomes were quantitatively analyzed. Serum levels of TNF- $\alpha$, IL- 6 , and IL- $1 \beta$ were measured by ELISA kit (R\&D Systems China) according to the manufacturer's instructions.

\section{Muscular toxicity evaluation}

We evaluated potential muscular toxicities of the amiloride treatment in the CT26 and LLC models using C57BL/6 and $\mathrm{BALB} / \mathrm{c}$ normal control mice, respectively. Either $12 \mathrm{C} 57 \mathrm{BL} / 6$ mice or $12 \mathrm{BALB} / \mathrm{c}$ mice were divided into 2 groups: NOR mice and NOR-AM mice, 6 per group. PBS was subcutaneously injected into the right flank of the NOR and NOR-AM mice on day 0 . From day 9, the NOR-AM mice were intraperitoneally injected daily with amiloride at the same dose of $2 \mathrm{mg} /$ $\mathrm{kg}$ following the procedure described above, whereas the NOR mice with PBS continually. Both the NOR-AM and the NOR mice were sacrificed on day 30 , and toxic effects of amiloride were assessed by the differences in body weight, gastrocnemius muscle weight, and plasma exosome density between the NOR-AM mice and the NOR mice.

\section{Histology study}

C2C12 myotubes were fixed with pre-cold methanol for $30 \mathrm{~s}$, stained with $0.1 \%$ crystal violet solution for $10 \mathrm{~min}$, and rinsed with distilled water before taking microscopic photographs. Myotube diameters were measured in a total of 200 myotubes from $\geq 10$ random fields. Mouse 
gastrocnemius was collected and fixed in 4\% PFA. Paraffin sections were stained with $H \& E$, and stained slides were assessed using phase-contrast microscopy. Myofiber areas were quantified by using ImageJ. To produce frequency distribution histograms, five view fields were measured with about 200 myofibers per field in each section.

\section{Protein expression analysis}

Proteins were extracted using RIPA lysis buffer containing protease inhibitor and phosphorylation protease inhibitor cocktails (Thermo Fisher, USA). The homogenates were then sonicated for $35 \mathrm{~s}$ and centrifuged $\left(11,000 \mathrm{~g}, 10 \mathrm{~min}, 4^{\circ} \mathrm{C}\right)$ to remove the debris. The supernatants were collected, and protein concentrations were determined by BCA Protein Assay Kit (Beyotime Biotechnology). Then, the denatured samples were subjected to SDS-PAGE and transferred to PVDF membranes (GE Healthcare, USA) for immunoblotting analysis. After blocking with 5\% non-fat milk in Trisbuffered saline containing $0.1 \%$ Tween 20 for $1 \mathrm{~h}$, the membranes were probed with corresponding antibodies. Proteins were visualized by enhanced chemiluminescence using horseradish peroxidase-conjugated antibodies and band densities were quantified by the ImageJ software.

\section{NMR-based metabolomic analysis}

Aqueous metabolites were extracted from mouse gastrocnemius for NMR-based metabolomic analysis according to the protocol described previously [18-20]. All NMR experiments were performed at $25^{\circ} \mathrm{C}$ on a Bruker Avance III $850 \mathrm{MHz}$ spectrometer (Bruker BioSpin, Germany) equipped with a TCI cryoprobe. Both the unsupervised principal component analysis (PCA) and supervised partial least-squares discriminant analysis (PLSDA) were applied to compare metabolic profiles of gastrocnemius among the four groups of the NOR, CAC, AM, and KD mice by using the SIMCA 14.1 software (MKS Umetrics AB, Sweden). The metabolic pathway analysis was performed to identify significantly altered metabolic pathways (significant pathways) on the MetaboAnalyst 4.0 webserver (https://www. metaboanalyst.ca). This webserver was also used to obtain heat-map plots of relative metabolite levels, and the Pearson's correlation coefficients between catabolic protein expressions and metabolite levels.

\section{Transcriptomic analysis}

The RNA-seq experiments were performed by Gene Denovo Biotechnology Co. (Guangzhou, China). The NOISeq R/Bioc package was used to identify differentially expressed genes (DEGs) from pairwise comparisons among the groups of mouse gastrocnemius with two criteria: fold change (FC) $\geq 1.5$ or $\mathrm{FC} \leq 0.67$, false discovery rate $(\mathrm{FDR}) \leq 0.05$. The Kyoto Encyclopedia of Genes and Genomes (KEGG) database was employed to conduct the pathway enrichment analysis based on the identified DEGs. Pathways with $\mathrm{Q}$ value $\leq 0.01$ were identified to be significantly changed biological processes.

\section{General statistical analysis}

Experimental data were expressed as means \pm SD. For the quantitative comparison between two groups, data were analyzed by Student's t test analysis using GraphPad Prism. For pairwise comparisons among three or more groups, data were analyzed by using one-way ANOVA followed by Tukey's multiple comparison test using the SPSS 19 software. Statistical significances were as follows: $p>0.05$ (NS), $p<0.05$ (*) $^{(*} \mathrm{p}<0.01$ (**) $^{(*)} p<$ $0.001{ }^{(* * * *)}$ ), and $p<0.0001\left(^{(* * * * *)}\right.$.

\section{Antibodies and reagents}

The detailed information about antibodies and reagents is displayed in Table S1.

\section{Results}

Amiloride treatment ameliorated cachectic muscle atrophy in mice

The processes of establishing mouse models are illustrated in Fig. S1. The CT26 and LLC cells or PBS were subcutaneously injected into the mice on day 0 , while amiloride or PBS was intraperitoneally injected daily from day 9 once mice developed palpable tumors. Both mouse body weights and tumor volumes were monitored along the process of establishing the CT26 cachexia model or the LLC cachexia model (Fig. 1a, b; Fig. 2a, b). On day 30 after tumor inoculation, the tumor-bearing mice displayed significant CAC features including decreases in body weights (mean loss rate: CT26, 5.9\%; LLC, 6.5\%), tumor-free body weights (TFBWs, mean loss rate: CT26, 14.8\%; LLC, 12.4\%), hind limb muscle weights (HLMWs, mean loss rate: CT26, 27.1\%; LLC, 26.2\%), gastrocnemius weights (mean loss rate: CT26, 33.1\%; LLC, 27.4\%), and soleus weights (mean loss rate: CT26, 26\%; LLC, 21\%) as well as muscle strengths compared to the NOR mice (Fig. 1d-h; Fig. 2d-h). Compared to the NOR mice, the CAC mice exhibited obvious myasthenia with the dramatic decline (CT26, 29.6\%; LLC, 27.1\%) in grip strength (Fig. 1 h; Fig. 2 h). In both the CT26 model and the LLC model, AM mice exhibited alleviated cachexia features as reflected by maintained body weights, TFBWs, HLMWs, and gastrocnemius weights as well as improved muscle strengths compared with the CAC mice. Besides, the amiloride treatment significantly increased soleus weights in the CT26 cachexia model, but not observably 


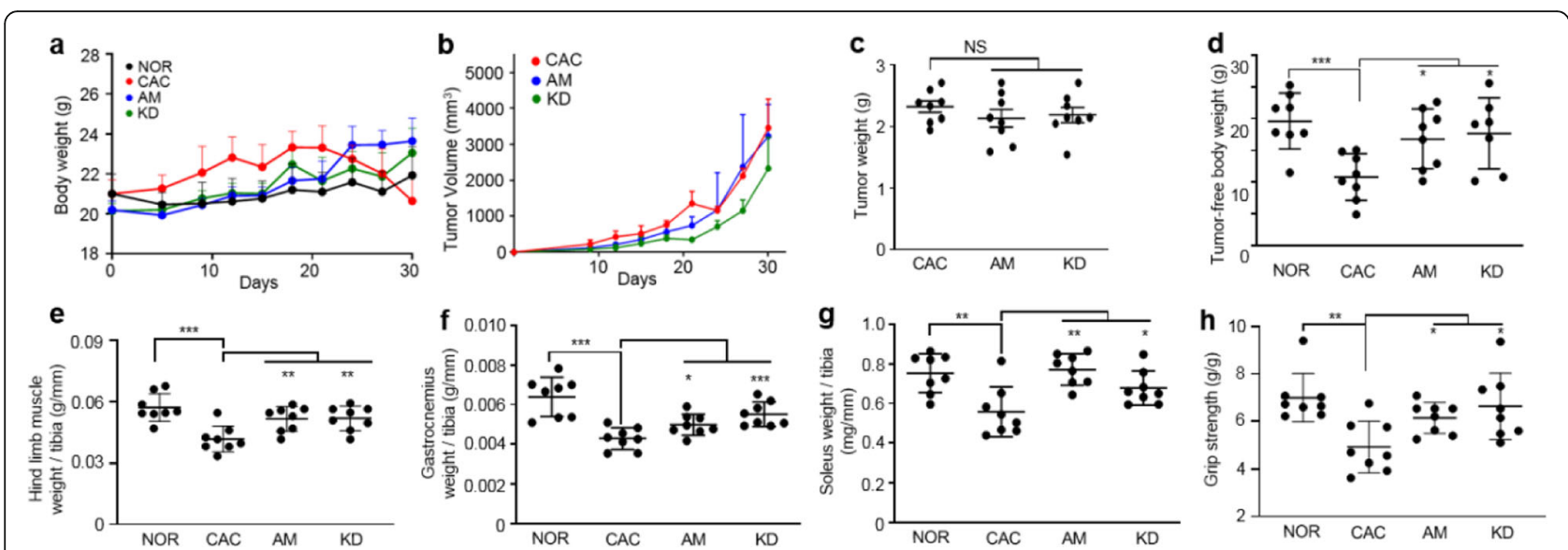

Fig. 1 Amiloride alleviated cachexia progression in the CT26 murine model. $\mathbf{a}, \mathbf{b}$ Body weight and tumor volume growth curves of the mice during the processes of establishing the animal models. $\mathbf{c}-\mathbf{h}$ Characterization of cachexia features in the mice on day 30 ( = 6-8). $\mathbf{c}$, $\mathbf{d}$ Tumor weights and tumor-free body weights of the mice. e Hindlimb muscle weights normalized to tibia length. $\mathbf{f}$ Gastrocnemius weights normalized to tibia length. $\mathbf{g}$ Soleus weights normalized to tibia length. $\mathbf{h}$ Grip strengths normalized to bodyweight. Statistical significances: $p>0.05$, NS; $p<$ 0.05 , *; $p<0.01$, **; $p<0.001$, ***. NOR, C57BL/6 or BALB/c normal control mice; CAC, CT26 cachexia mice; AM, amiloride-treated mice; KD, mice inoculated with Rab27-knockdown CT26 cells

changed those in the LLC cachexia model (Fig. 1; Fig. 2). The cross-section area analysis revealed a decreased myofiber size in gastrocnemius of the CAC mice (cachectic gastrocnemius), indicating the muscle dysfunction condition in the CT26 and CT26 cachexia models (Fig. 3a-d). As is known, skeletal muscle mass is influenced by the counter-balance between muscle degradation and myogenesis. In cachectic gastrocnemius, the decreased ratio of p-FoxO3a/FoxO3a (Fig. S2) distinctly upregulated the expressions of Atrogin-1 and MuRF-1 (Fig. 3e, f). The amiloride treatment significantly downregulated the levels of Atrogin-1 and MuRF-1 in gastrocnemius (Fig. 3e, f).
Interestingly, expressions of MyoD1 and Myogenin were not statistically significantly changed both of which regulated muscle cell growth and differentiation (Fig. 3e, f). In general, the amiloride treatment preserved muscle weights and muscle strengths in tumor-bearing mice.

\section{Amiloride treatment alleviated cachexia-related features in mice}

Cachexia is usually accompanied by fat loss. We analyzed epididymal adipose tissues in the CT26 model and the LLC model, especially white adipose tissues which are responsible for the organismal energy storage.

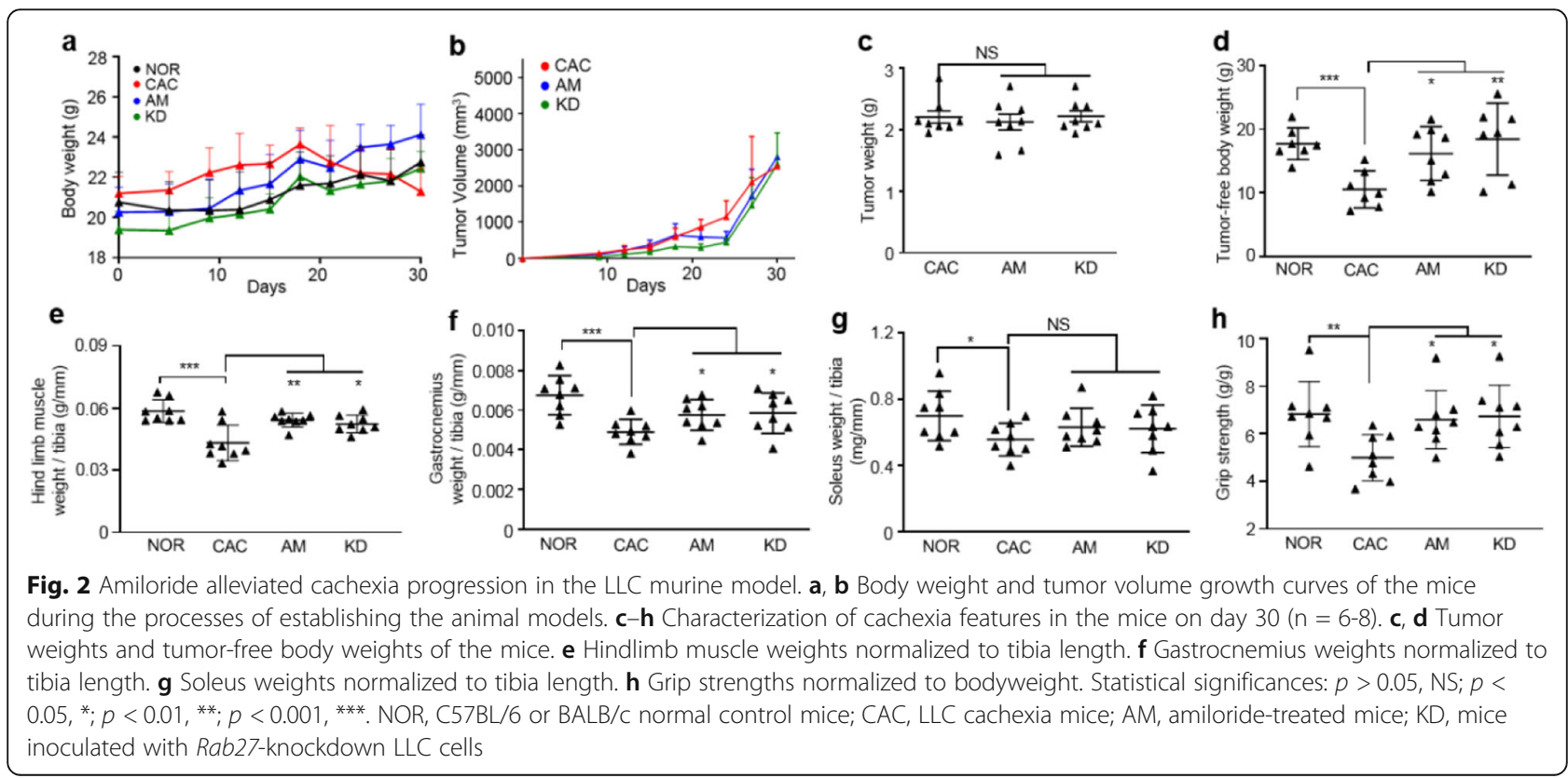




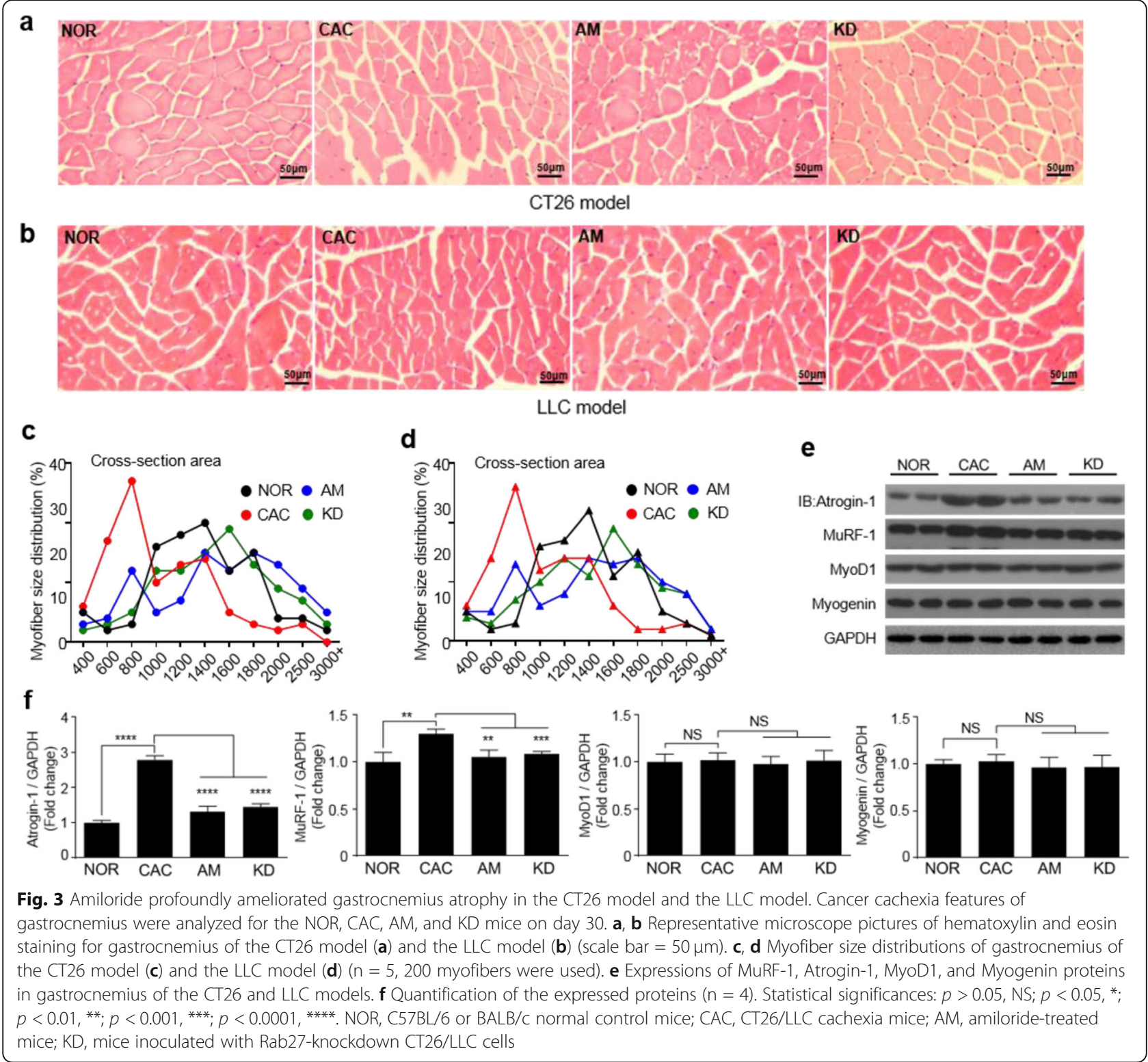

Compared with the NOR mice, the CAC mice displayed observable loss of epididymal adipose tissues, which was partially restored in the AM mice (Fig. 3a, b; Fig. 4a, b).

On the other hand, cachexia is mostly associated with systemic inflammatory response. In both the CT26 model and the LLC model, the CAC mice showed a profoundly upregulated serum level of IL- 6 and almost identical serum levels of TNF- $\alpha$ and IL-1 $\beta$ compared with the NOR mice (Fig. S3c-e; Fig. S4c-e). Remarkably, the AM mice displayed declined serum level of IL- 6 but nearly unchanged serum levels of TNF- $\alpha$ and IL-1 $\beta$ compared to the CAC mice in both models (Fig. S3c-e; Fig. S4c-e). Furthermore, the expression of down-stream phosphorylated Stat3 was remarkably upregulated in gastrocnemius of the CAC mice (CAC gastrocnemius), corresponding to the profoundly upregulated serum IL-6 level. Significantly, the expression of phosphorylated Stat3 was downregulated in gastrocnemius of the AM mice (AM gastrocnemius; Fig. S2). In addition, given that the p38 kinase participates in the cellular response to multiple stresses and inflammatory cytokines, we analyzed expressions of phosphorylated p38 and cleaved caspase-3, and observed significant enhancements of p38 kinase and caspase- 3 activities in the CAC gastrocnemius, which was partially reversed in the AM gastrocnemius (Fig. S2).

Considering that anorexia is a crucial factor of cachexia, we continuously monitored food intakes across the four groups of mice. In the CT26 model, the CAC mice showed a slight decrease in average food intake 


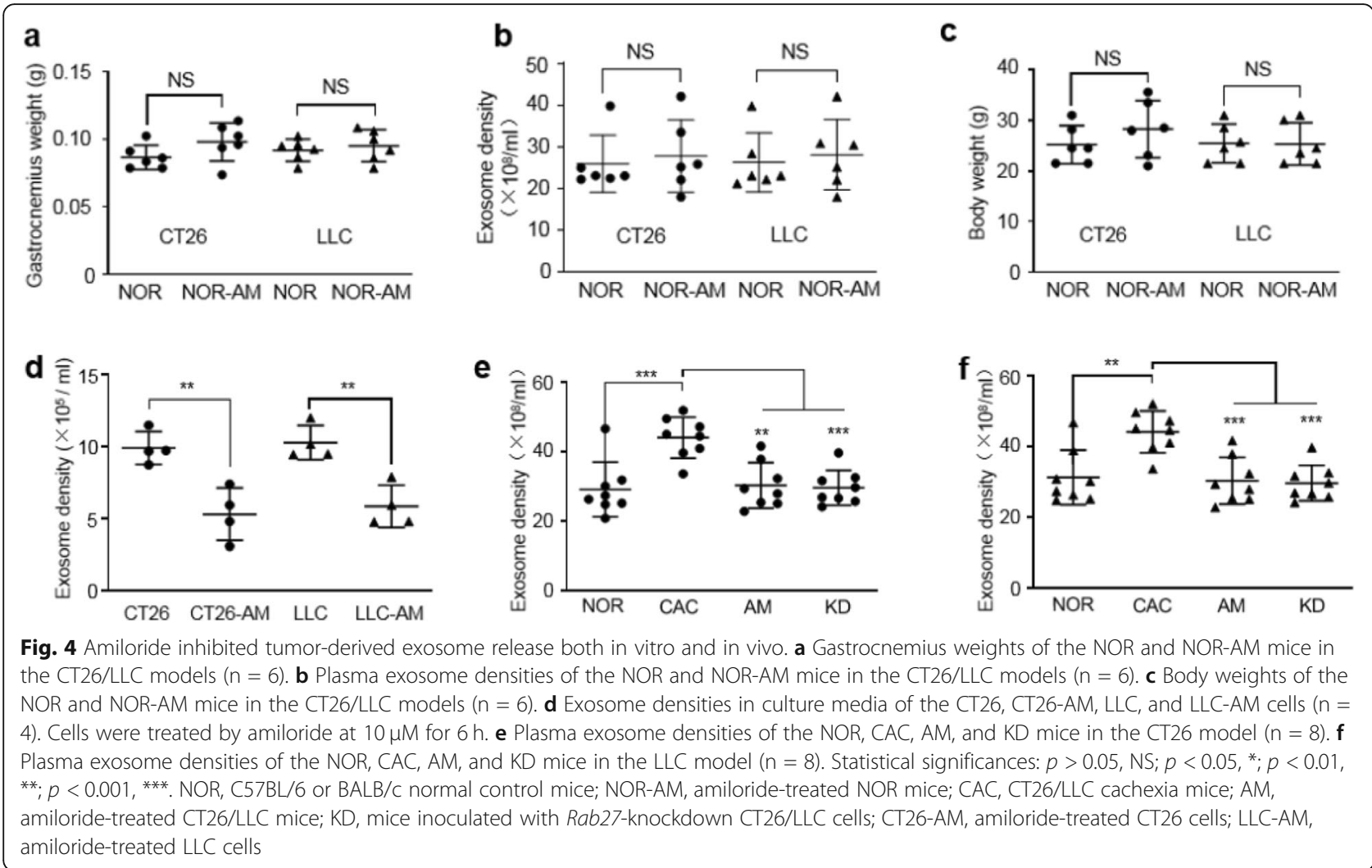

compared with the other three groups of mice (Fig. S3f). Differently, in the LLC model, the CAC mice showed an average food intake similar to the other three groups of mice (Fig. S4f). Besides, both CT26 and LLC models did not exhibit observable differences in heart weight among the four groups of mice (Fig. S3g; Fig. S4g).

Amiloride treatment ameliorated muscle wasting through inhibiting tumor-derived exosome release

We further investigated molecular mechanisms underlying the favorable anti-cachexia effects of the amiloride treatment. The AM mice exhibited similar tumor weights to the CAC mice in both the CT26 model and LLC model (Fig. 1c; Fig. 2c). Furthermore, the weight of the NOR-AM gastrocnemius did not show statistically significant change relative to the NOR gastrocnemius in both models (Fig. 4a).

Given that amiloride can exert inhibitory effects on the cellular exosome release [12, 13], we treated the CT26 and LLC cells with amiloride at various concentrations $(1-200 \mu \mathrm{M})$ for $6 \mathrm{~h}$ (plasma half-life of amiloride is about 6-9h) and did not observe statistically significant changes in the viabilities of both tumor cells (Fig. S5). We isolated exosomes from culture media of the CT26 cells, CT26-AM cells, LLC cells, and LLC-AM cells and characterized the four groups of tumor-derived exosomes (CT26 exosomes, CT26-AM exosomes, LLC exosomes, LLC-AM exosomes). Significantly, the treatment of amiloride at $10 \mu \mathrm{M}$ for $6 \mathrm{~h}$ evidently inhibited exosome release from the CT26 and LLC tumor cells (Fig. 4d), indicating that the amiloride treatment profoundly decreased exosomes produced by the tumor cells.

Furthermore, we isolated exosomes from plasma of the four groups of mice in the CT26 model and the LLC model and quantified plasma exosome densities. Notably, the CAC mice showed dramatically increased plasma exosome densities compared to the NOR mice in both models (Fig. 4e, f). More importantly, the AM mice exhibited distinctly reduced plasma exosome densities in the two cachexia models (Fig. 4e, f), indicating that the amiloride treatment efficiently inhibited tumor-derived exosome release. Note that the amiloride treatment did not significantly influence the normal tissue-derived exosome release as indicated by the statistical comparison of plasma exosome density between the NOR-AM mice and the NOR mice (Fig. 4b). Besides, the NOR-AM mice did not show observable muscular toxicities as reflected by basic unchanged body weights and gastrocnemius weights compared to the NOR mice (Fig. 4a, c).

We isolated and characterized exosomes from plasma of the CAC patients and the Non-CAC patients (CAC exosomes, non-CAC exosome; Fig. S6-8). Based on the limited patient samples used in this study, we found that 
exosome densities in patient plasma varied even by 20 fold among individuals (Fig. S8b). In addition, the CAC patients did not display significant statistical differences in plasma exosome density from the Non-CAC patients (Fig. S8b).

On the other hand, the exosomes derived from both the CT26/LLC culture media and the CAC patient plasma induced apparent myotube atrophy in vitro, as indicated by the obviously decreased myotube diameters (Fig. S8a-d, h-i) and distinctly enhanced expressions of Atrogin-1 and MuRF-1 relative to PBS controls (Fig. S9). Our observation that plasma exosomes of the CAC patients profoundly promoted myotube atrophy, supporting the previous studies $[7,21]$.

To further confirm the experimental observation that tumor-derived exosomes significantly induced muscle atrophy in cancer cachexia, we constructed Rab27 knock-down CT26 and LLC cell lines (CT26-KD cells, LLC-KD cells) as positive controls (Fig. S8e, f). Expectedly, knock-down of Rab27a and Rab27b potently inhibited exosome release from tumor cells without remarkably changing viabilities of the tumor cells (Fig. S8g; Fig. S10). The exosomes isolated from culture media of the CT26-KD and LLC-KD tumor cells displayed markedly reduced abilities to induce myotube atrophy relative to their corresponding wild-type counterparts (Fig. S8h, i; Fig. S9b). As expected, the KD mice inoculated with the Rab27 knock-down tumor cells displayed alleviated cachexia features, as indicated by increased body weights, TFBWs, HLMWs, and gastrocnemius weights as well as improved muscle strengths compared with the CAC mice in the two models (Fig. 1; Fig. 2). Furthermore, the CAC, AM, and KD mice of both models showed similar tumor weights on day 30 (Fig. 1c; Fig. 2c), indicating that amiloride inhibited tumor-derived exosome release without significantly affecting tumor growth in vivo. These results indicated that the amiloride treatment could profoundly ameliorate cachectic gastrocnemius atrophy and thereby alleviate cancer cachexia through inhibiting tumor-derived exosome release.

\section{Amiloride treatment improved hyper-catabolism in gastrocnemius}

To mechanistically understand metabolomic features of skeletal muscles in the four groups of mice, we conducted NMR-based metabolic profiling of gastrocnemius. A total of 32 metabolites were identified (Fig. S11a; Table S2). The resonance assignments were confirmed by using $2 \mathrm{D}$ ${ }^{1} \mathrm{H}-_{-}{ }^{13} \mathrm{C}$ HSQC spectra (Fig. S11b).

The unsupervised principal component analysis (PCA) scores plot illustrates that the metabolic profile of the CAC gastrocnemius is distinctly different from those of the NOR, AM and KD gastrocnemius (Fig. 5a). Similarly, the supervised partial least-squares discriminant analysis (PLS-DA) scores plots display significant metabolic distinctions in gastrocnemius between the NOR and CAC mice, the CAC and AM mice, and the CAC and KD mice (Fig. 5b). Significant metabolites were identified from the PLS-DA models (Fig. S12). Furthermore, we performed univariate analysis to quantitatively compare metabolite levels among the four groups of gastrocnemius and identify differential metabolites (Table S3).

Combining the significant metabolites with the differential metabolites, we determined characteristic metabolites for the four groups of mouse gastrocnemius (Table S4). Totally, 18, 20, and 22 characteristic metabolites were identified in gastrocnemius for CAC vs. NOR, AM vs. CAC, and KD vs. CAC, respectively. Among 17 shared characteristic metabolites, 10 metabolites (glucose, IMP, glycine, creatine, methylmalonate, niacinamide, aspartate, glutamate, fumarate and tyrosine) were increased in the CAC gastrocnemius but consistently decreased in the AM and KD gastrocnemius, and 7 metabolites (lactate, taurine, inosine, alanine, 2-phosphoglycerate, ATP and glutamine) were decreased in the CAC gastrocnemius but consistently increased in the AM and KD gastrocnemius (Table S4).

Furthermore, significant pathways were identified by performing the metabolic pathway analysis for the four groups of gastrocnemius (Fig. 5c). Notably, both the amiloride treatment and Rab27 knock-down significantly altered the identical metabolic pathways. Interestingly, 8 of the 11 significant pathways were closely related to amino acid metabolism. The heat-map plot of relative metabolite levels illustrates that a large number of amino acids were increased in the CAC gastrocnemius, including alanine, glutamine, aspartate, and glycine (Fig. 6a). The upregulated levels of phosphorylated AMPK-Tyr112 and downregulated levels of phosphorylated Akt1 indicated a strongly activated catabolism in cachectic gastrocnemius (Fig. 6b, c). Moreover, the raised ratio of LC3II/ LC3I and declined levels of MHC and MLC were indicative of promoted autophagy in cachectic gastrocnemius (Fig. 6b, c). Additionally, the levels of upregulated amino acids were positively correlated with expressions of catabolic proteins, but negatively correlated with expressions of anabolic proteins in the CAC gastrocnemius (Fig. 6d).

Transcriptomic profiling identified 1466 upregulated genes and 1941 downregulated genes in the CAC gastrocnemius relative to the NOR gastrocnemius, of which 753 differentially expressed genes (DEGs) were shared by the pairwise comparisons of CAC vs. NOR, AM vs. CAC, and KD vs. CAC (Fig. S13a; Fig. S14a). The KEGG enrichment analysis based on the identified DEGs screened out distinctly changed metabolism-related processes including 


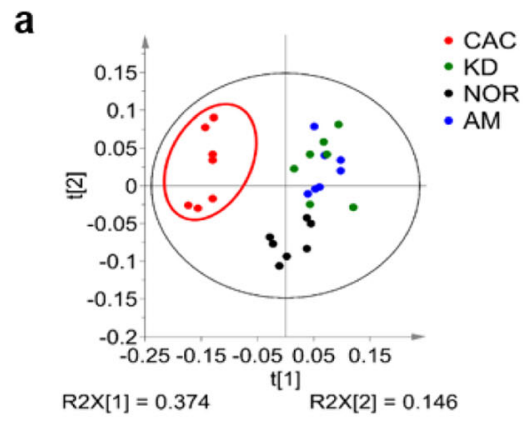

b
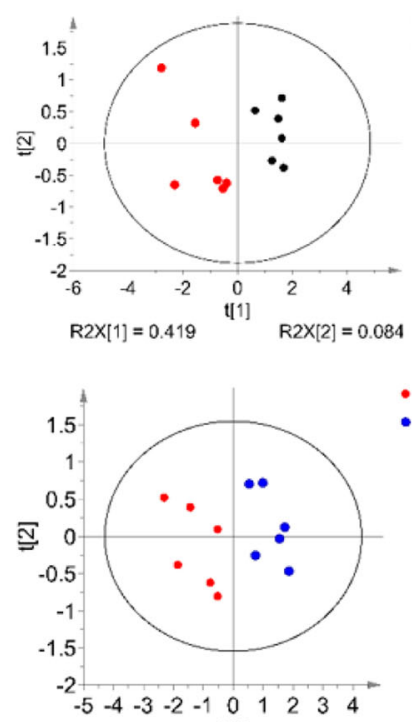

\section{- $\mathrm{CAC}$}

- AM

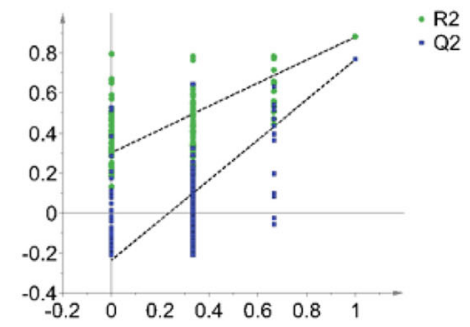

$\operatorname{R} 2 \times[1]=0.733$

t[1]

$R 2 X[2]=0.124$

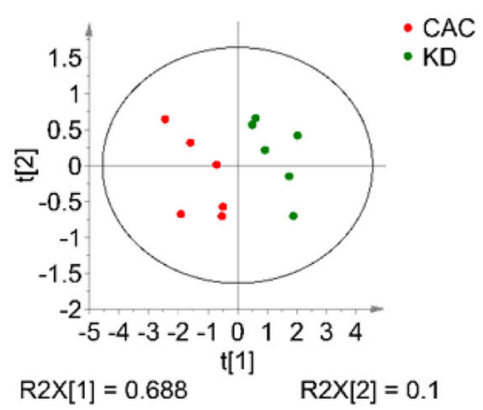

C
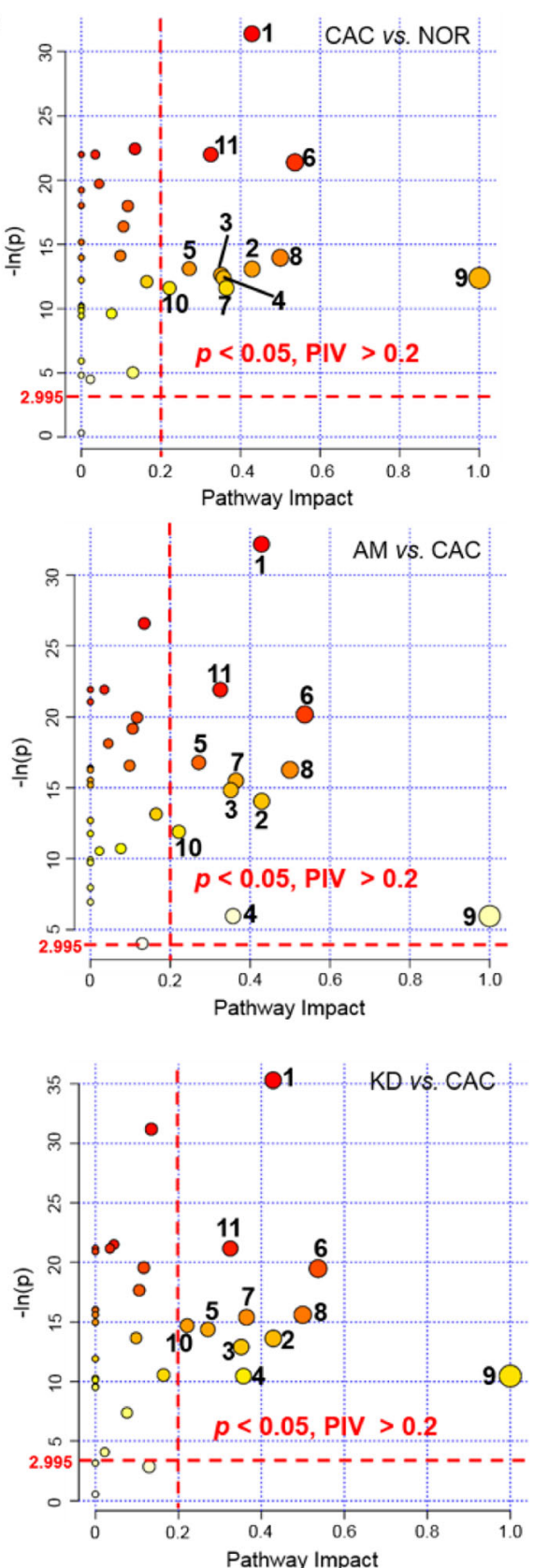

Fig. 5 Metabolomic analyses of the NOR, CAC, AM and KD gastrocnemius. a PCA scores plot of 1D ${ }^{1} \mathrm{H}$ NMR data obtained on aqueous extracts derived from gastrocnemius of the NOR, CAC, AM and KD mice $(n=7)$. $\mathbf{b}$ PLS-DA scores plots and cross-validation plots of $1 \mathrm{D}^{1} \mathrm{H}$-NMR data obtained from gastrocnemius of the NOR, CAC, AM and KD mice. Top: CAC mice vs. NOR mice; Middle: AM mice vs. CAC mice; Bottom: KD mice vs. CAC mice. The PLS-DA models were cross-validated to evaluate their robustness with random permutation tests (200 cycles). c Metabolic pathway analyses of CAC vs. NOR, AM vs. CAC, KD vs. CAC, using the Pathway Analysis module provided by MetaboAnalyst 4.0. Numbers in the three panels represent significantly altered metabolic pathways, which were identified with $-\ln (p)>2.995$ (corresponding to $p<0.05$ ) and pathway impact value $>0.2: 1$, taurine and hypotaurine metabolism; 2 , nicotinate and nicotinamide metabolism; 3 , pyruvate metabolism; 4 , phenylalanine metabolism; 5, glycine, serine and threonine metabolism; 6, alanine, aspartate and glutamate metabolism; 7, glutathione metabolism; 8, D-glutamine and D-glutamate metabolism; 9, phenylalanine, tyrosine and tryptophan biosynthesis; 10, histidine metabolism; 11, starch and sucrose metabolism. NOR, C57BL/6 or BALB/c normal control mice; CAC, CT26/LLC cachexia mice; AM, amiloride-treated mice; KD, mice inoculated with Rab27-knockdown CT26/LLC cells 


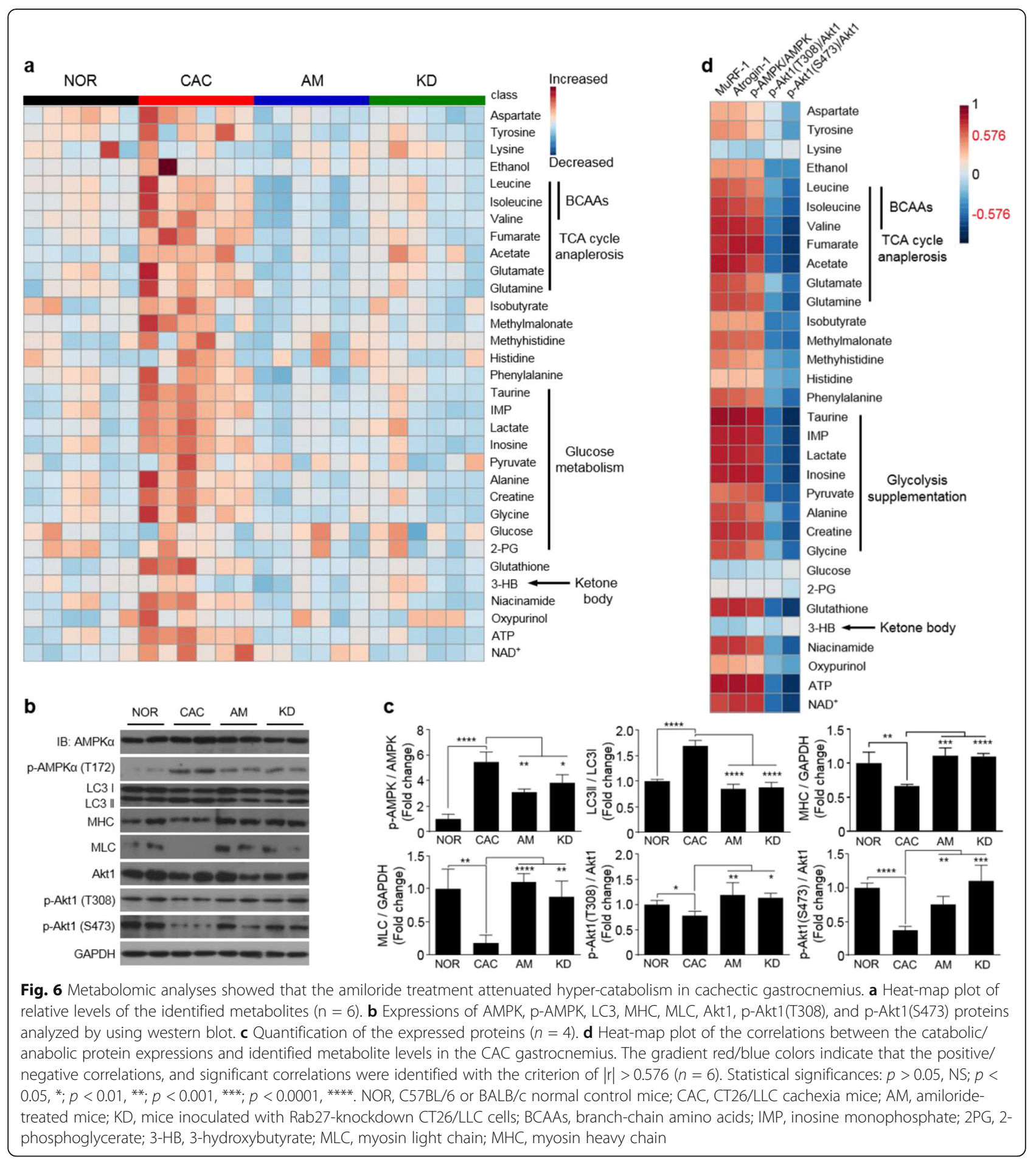

carbohydrate metabolism, lipid metabolism, and amino acid metabolism (Fig. S13b; Fig. S14b). Consistently, the loss of epididymal adipose tissues in cachexia mice (Fig. S2a, b; Fig. S3a, b) was accompanied by upregulated expressions of fatty acid translocase CD36 and Acyl-coenzyme A thioesterase $1(A \cot 1)$ in the CAC gastrocnemius (Fig. 7b, c; Table S5), which facilitated mobilization and oxidation of adipose tissues.

Overall, the amiloride treatment substantially improved hyper-catabolism in cachectic gastrocnemius, and $R a b 27$ knock-down showed a similar improvement of hyper-catabolism (Fig. 6; Fig. 7). 

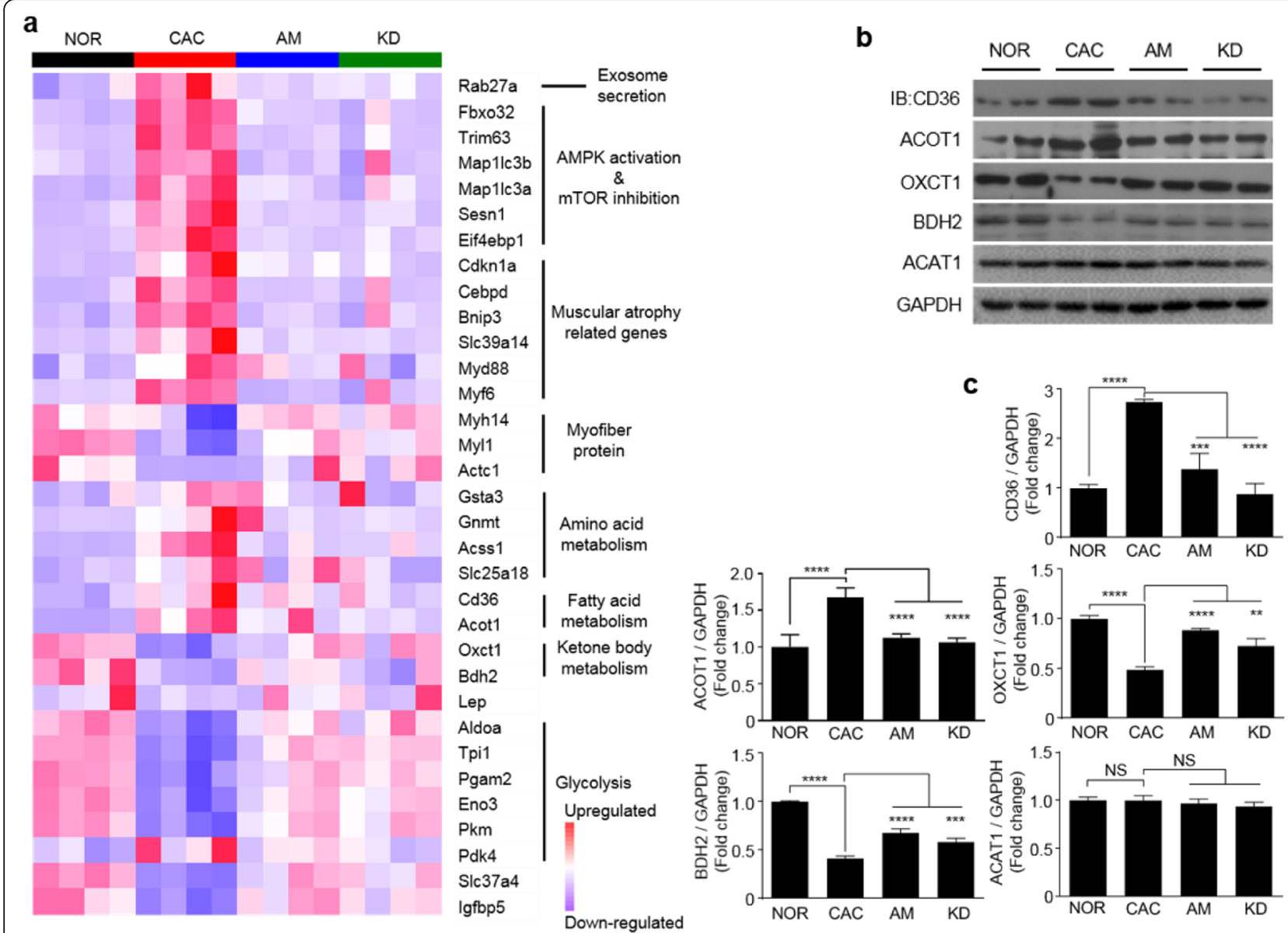

Fig. 7 Transcriptomic analyses exhibited that the amiloride treatment improved glycolysis and ketone body oxidation in cachetic gastrocnemius. a Heat-map plot of relative transcription levels of muscular atrophy-related genes $(n=4)$. $\mathbf{b}$ Expressions of ACOT1, CD36, OXCT1, BDH2, and ACAT1 proteins. c Quantification of the expressed proteins $(n=4)$. Statistical significances: $p>0.05, \mathrm{NS} ; p<0.05, * ; p<0.01,{ }^{* *} ; p<0.001$, ***; $p<$ $0.0001, * * *$. NOR, C57BL/6 or BALB/c normal control mice; CAC, CT26/LLC cachexia mice; AM, amiloride-treated mice; KD, mice inoculated with Rab27-knockdown CT26/LLC cells; ACOT1, acyl-coenzyme A thioesterase 1; CD36, fatty acid translocase; OXCT1, 3-oxoacid CoA transferase 1; $\mathrm{BDH} 2$, 3-hydroxybutyrate dehydrogenase 2; ACAT1, acetyl coenzyme A acetyltransferase 1

\section{Amiloride treatment improved blocked glycolysis and} impeded ketone body oxidation in gastrocnemius

Glycolysis is one of the fundamental energy sources in muscle cells, and ketone bodies are alternative energy sources under harsh conditions. Glucose was significantly increased in the CAC gastrocnemius relative to the NOR gastrocnemius, contrarily, the glycolysis end product-pyruvate-was decreased (Table S3). The expressions of multiple glycolytic catalyzing enzymes were downregulated, but the expression of glycolytic inhibition enzyme (pyruvate dehydrogenase kinase isoenzyme 4, $P d k 4$ ) was upregulated in cachectic gastrocnemius relative to normal control (Fig. 7a; Table S5). Furthermore, the CAC gastrocnemius exhibited more than 2fold decreases in expressions of ketone body oxidation enzymes 3-oxoacid CoA transferase 1 (OXCT1) and 3hydroxybutyrate dehydrogenase 2 (BDH2; Fig. 7b,c) but substantially unchanged expressions of ketone body transporters (MCT1 and MCT4; Fig. S2) and the dominant organismal ketone body-3-hydroxybutyrate (3-HB; Table S3). These results were indicative of blocked glycolysis and impeded ketone body oxidation in the CAC gastrocnemius. Significantly, both the AM and KD gastrocnemius displayed profoundly improved glycolysis and ketone body oxidation via the inhibition of tumorderived exosome release (Fig. 7; Fig. S15).

\section{Discussion}

Cancer cachexia evidently reduces patient survival and quality of life due to its high incidence and mortality rate $[4,22]$. Developments of efficient therapeutic strategies are urgently required for the CAC treatments. Previous studies have demonstrated that amiloride possesses potassium-sparing diuretic function, which has been clinically used in the treatments of hypertension and edema due to heart failure [11, 23-25]. Moreover, 
amiloride can inhibit exosome release from cells and reverse exosome-promoted pathogenic processes [26, 27]. In this study, we established CT26/LLC-induced mouse models of lung/colorectal cancer cachexia, assessed the effects of the amiloride treatment for alleviating muscle atrophy in the two cachexia models, and addressed the underlying molecular mechanisms. Our results reveal that amiloride is a potential therapeutic drug capable of ameliorating muscle wasting in cancer cachexia through inhibiting tumor-derived exosome release (Fig. 8).

Both the CT26 model and the LLC models showed remarkable cachexia features and significant metabolic impairments in gastrocnemius. Significantly, the amiloride treatment prevented the losses of body weight, skeletal muscle, and fat mass, which did not obviously affect tumor growth and induce observable systemic toxicities in normal control mice, as indicated by basically unchanged body weights and gastrocnemius weights of the NOR-AM mice relative to the NOR mice. More importantly, multiple cachexia features were improved, as evidenced by downregulated expressions of muscular atrophic proteins, partially restored muscle strength and neutralized systemic inflammation. The further mechanistic study revealed that the amiloride treatment profoundly inhibited tumor-derived exosome release and attenuated hyper-catabolism, significantly improved the metabolic impairments in cachectic gastrocnemius, thereby alleviating the CAC progression.

By integrative metabolomic and transcriptomic analyses, we identified significantly impaired metabolic pathways in cachectic gastrocnemius relative to normal control, including promoted muscular catabolism, inhibited muscular protein synthesis, blocked glycolysis, and impeded ketone body oxidation. Expectedly, the impaired metabolic pathways potentially contribute to metabolic mechanisms underlying the CAC progression. Previously, we established a mouse model of gastric cancer cachexia by orthotopically implanting BGC 823 cells and identified significantly impaired metabolic pathways in cachectic gastrocnemius [19]. The two works of ours unanimously confirm that aberrant catabolism in cachectic gastrocnemius is triggered primarily by upregulated E3 ligases, which exhibit profoundly increased levels of amino acids (isoleucine, leucine, valine, glutamate). Interestingly, the CT26/LLC cachexia models show blocked glycolysis in gastrocnemius, while the BGC 823 cachexia model displays promoted glycolysis. The metabolic distinction might be dictated by several factors including differences in food intake, inflammatory cytokines, tumor type, and stage of cachexia. Additionally, the discrepancy between orthotopic and subcutaneous cachexia models might be associated with discrepant metabolic features in gastrocnemius, potentially contributing to the metabolic distinction, even though these models undergo similar muscular atrophic processes.

In the past decades, strategies for cachexia therapy mainly focused on the development of new drugs, including ghrelin and ghrelin receptor agonists, myostatin antagonists, inflammatory cytokine neutralizing antibodies, and natural product extracts [28-37]. Furthermore, recent studies have identified several key molecules (namely cachectin) and correspondingly explored inhibitory chemicals for alleviating the CAC progression. However, most of these efforts have not obtained satisfactory clinical trial results [38]. The primary reason might attribute to the heterogeneity of cachectin resulting from either different tumor types or being aroused by inherent reprogramming processes within identical tumor types, which requires more comprehensive investigation. Besides, it is time-consuming

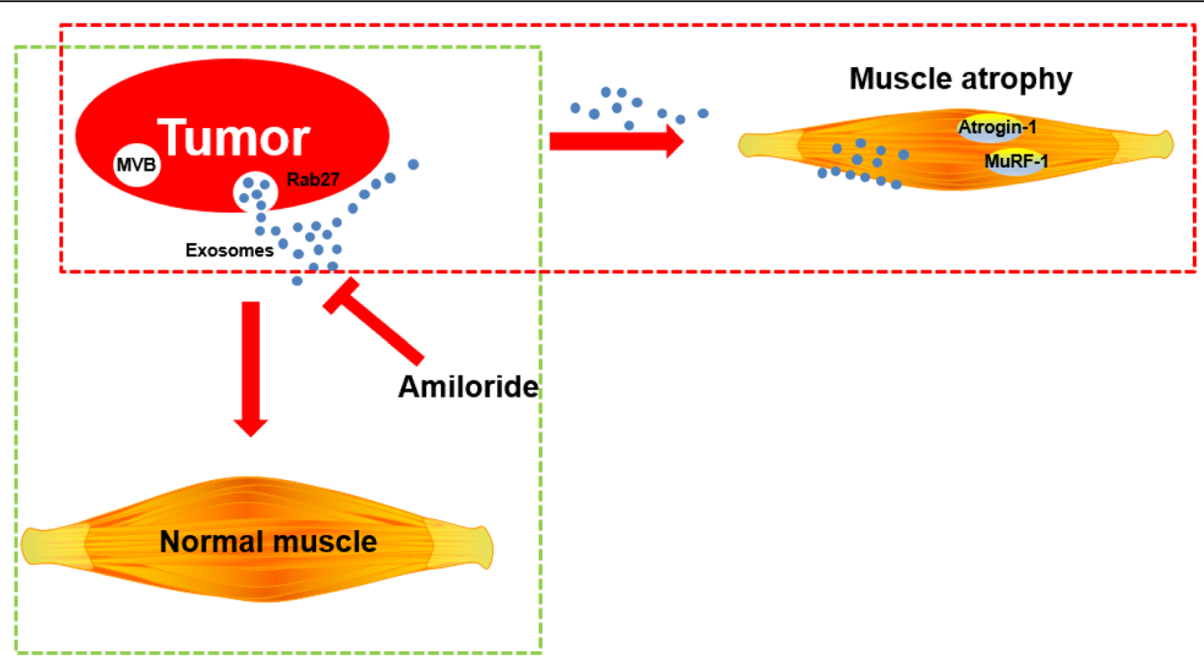

Fig. 8 Graphic model of the amiloride treatment ameliorating cachectic muscle wasting through inhibiting tumor-derived exosome release 
for clinical trials and further approval of newly developed drugs. In contrast, applying existing drugs for new indications could be a more feasible alternative strategy. Given that most of the existing drugs are usually associated with well addressed pharmacokinetic and pharmacodynamic properties and toxicity profiles, the alterative strategy might greatly reduce the time required to develop novel drugs for the CAC treatment.

Amiloride has been clinically used for nearly three decades in the treatments of hypertension, edema and congestive heart failure [23-25, 39]. In the present study, we demonstrated for the first time the therapeutic potentials of amiloride in the treatments of cancer cachexia. Previously, Cameron et al. showed that increased $\mathrm{Na}^{+}$contents in multiple tissues of $\mathrm{H} 6$ hepatoma-induced cachexia mice can be partially reversed by the amiloride treatment [40]. Their study mainly determined amiloride-induced changes of $\mathrm{Na}^{+}$and other ions contents in liver cachexia mice, but did not further examine whether cachexia symptoms were relieved.

The most outstanding characteristic of amiloride is the efficient inhibitory effects on the $\mathrm{Na}^{+} / \mathrm{H}^{+}$and $\mathrm{Na}^{+} / \mathrm{K}^{+}$ transporters [11, 23, 26, 41]. Under normal conditions, the $\mathrm{Na}^{+} / \mathrm{H}^{+}$transporter mediates $\mathrm{H}^{+}$efflux from the cells in exchange for $\mathrm{Na}^{+}$influx. Further works indicated that blocking $\mathrm{Na}^{+}$influx/ $\mathrm{H}^{+}$efflux can inhibit cell growth, and tumor cells are more vulnerable to the blocking of $\mathrm{H}^{+}$efflux than normal cells which might endow amiloride antineoplastic effects [26, 27, 41-44].

In this study, we observed that the treatment of amiloride at various concentrations $(1-200 \mu \mathrm{M})$ for $6 \mathrm{~h}$ did not observably change cell viabilities of tumor cells Moreover, both the CT26 model and the LLC model showed that the amiloride treatment did not statistically significantly affect tumor growth in the CAC mice. These results suggest that the antineoplastic effects of amiloride do not significantly contribute to amiloridemediated alleviation of the CAC progression.

On the other hand, intracellular $\mathrm{Na}^{+}$contents also regulate the trafficking of extracellular vesicles including exosomes [45]. The $\mathrm{Na}^{+} / \mathrm{Ca}^{2+}$ antiporter mediates the appropriate $\mathrm{Na}^{+}$efflux in exchange for $\mathrm{Ca}^{2+}$ influx. The increased cytoplasmic $\mathrm{Ca}^{2+}$ content is a prerequisite for multi-vesicular bodies (MVBs) generation and following exosome biogenesis [40, 45]. Thus, amiloride probably decreases intracellular $\mathrm{Ca}^{2+}$ content through mediating $\mathrm{Na}^{+}$efflux by the antiporter, and obstructs cellular exosome release. Note that we could not currently confirm this speculation as this study had not measured the contents of $\mathrm{Ca}^{2+}$ and $\mathrm{Na}^{+}$.

Both the CL26 and LLC murine models showed dramatic decreases in plasma exosome densities of the AM mice relative to the CAC mice. Note that plasma exosomes could also be derived from normal tissues besides tumor tissues. The amiloride treatment $(2 \mathrm{mg} / \mathrm{kg} /$ day $)$ did not statistically change plasma exosome densities derived from normal tissues in the normal control mice, as indicated by the observation that the NOR-AM mice and the NOR mice did not show statistically significant difference in plasma exosome density. Thus, it could be expected that the decreases in plasma exosome density in the amiloride-treated CAC mice might reflect the reductions in exosomes produced by the tumor cells. Furthermore, the quantitative analysis of exosome densities in culture media of the CT26/LLC tumor cells indicated that the amiloride treatment $(10 \mu \mathrm{M}, 6 \mathrm{~h})$ profoundly deceased exosome release from the tumor cells but not observably affected viabilities of the tumor cells. These experimental results reveal that the amiloride treatment significantly reduces exosome production by the tumor cells. Nevertheless, more experiments should be performed to further support the conclusion that amiloride ameliorates muscle wasting through inhibiting tumorderived exosome release and thereby alleviates cancer cachexia. Potentially, the alleviation of cachexia could be due to several factors including reduced circulating IL-6, maintenance of cardiac function, and direct effects on skeletal muscle, etc. These factors are worthy of further exploration in future studies.

A previous study exhibited that the amiloride treatment at two doses of $10 \mathrm{mg} / \mathrm{kg}$ and $15 \mathrm{mg} / \mathrm{kg}$ did not exhibit significant toxic effects in a multiple myeloma xenograft murine model, as indicated by basically unchanged body weights [27]. Consistently, the amiloride treatment at $2 \mathrm{mg} / \mathrm{kg}$ used in our study did not show observable muscular toxic effects, as indicated by basically unchanged body weights and gastrocnemius weights in the NOR-AM mice relative to the NOR mice. However, our limited results only reflect that amiloride has not significant muscle toxicity in healthy mice. A systemic toxicity test should be conducted in further studies. Nevertheless, the amiloride treatment may be beneficial to the amelioration of cachectic muscle wasting and thus to the alleviation of the CAC progression.

Previous studies documented that cancer cachexia can be induced by multiple factors [35, 38, 46, 47], including cytokines, hormones, tumor factors, and gut microbes. More significantly, the present study displayed that the exosomes isolated from plasma of cancer cachexia patients and culture media of the CT26/LLC tumor cells induced remarkable myotube atrophy, well confirming that tumor-derived exosomes can induce muscle wasting in cancer cachexia. Furthermore, it has been demonstrated that several individual components in tumorderived exosomes can significantly contribute to cancer cachexia progression, such as miR-21 [14], heat shock proteins [9], and metal ions [48]. Expectedly, exploration of other key components in tumor-derived exosomes 
would be greatly beneficial to further understand the molecular mechanisms of exosome-induced muscle wasting and early diagnosis of cancer cachexia. Such studies are worthy of being conducted in the future.

The present study revealed that the amiloride treatment can significantly inhibit tumor-derived exosome release, and thereby profoundly ameliorate muscle wasting and alleviate the CAC progression, indicating clinical potentials of amiloride for treatments of the CAC patients. Similar to amiloride, some other drugs or chemical inhibitors, such as GW4869 [49-51], omeprazole [12], chlorpromazine [52], and statins [53-55], also possess the effects of inhibiting cellular exosome release, and could be exploited as potential drugs against cancer cachexia too.

Cachectic muscle atrophy primarily results from an imbalance of catabolism and anabolism [2]. Amiloridemediated inhibition of tumor-derived exosome release significantly improves metabolic impairments in cachectic gastrocnemius. As is known, both activation of the AMPK signaling cascade and inhibition of the Akt pathway are responsible for muscle wasting in cancer cachexia. Moreover, activation of the apoptosis pathway mediated by the p38 kinase also contributes to myofibrillar protein degradation and muscle dysfunction. Cachectic gastrocnemius exhibited a more than 5 -fold increase in the ratio of p-AMPK (Tyr112)/AMPK and significant decreases in the ratio of $\mathrm{p}$-Akt1/Akt1, indicating the promoted catabolism and inhibited anabolism in cancer cachexia. The metabonomic analysis of the CAC gastrocnemius exhibited upregulated amino acid levels relative to normal control, further confirming the promoted degradations and inhibited syntheses of muscular proteins. The accumulated amino acids are capable of acting as supplementary sources for both TCA cycle anaplerosis and glycolysis, fulfilling increased energy demand in cancer cachexia. Furthermore, the transcriptomic analysis of the CAC gastrocnemius showed downregulated expressions of five glycolytic catalyzing enzymes and the upregulated expression of a glycolytic inhibiting enzyme relative to the NOR gastrocnemius, indicating that blocked glycolysis significantly promoted muscle wasting in cancer cachexia. More significantly, amiloride-mediated inhibition of tumor-derived exosome release enhances the promoted muscular proteolysis, inhibited muscular protein synthesis, and blocked glycolysis in cachectic gastrocnemius.

In addition, we found that tumor-derived exosomes are also involved in the regulation of ketone body metabolism in skeletal muscle. In harsh energy conditions (continuous intensive exercise training; constant hunger, etc.), ketone bodies are mostly transported across the blood-brain barrier to fuel the brain. In cachexia mice, hepatogenic ketone bodies are available energy sources for extrahepatic tissues (mainly in the brain and skeletal muscles). We detected downregulated expressions of key enzymes (BDH2 and OXCT1) for ketone body oxidation in cachectic gastrocnemius, implying that ketone body oxidation was potentially impeded. Consistently, the inhibition of tumor-derived exosome release by the amiloride treatment can alleviate the impediment of ketone body oxidation in cachectic gastrocnemius. We thus speculate that the impeded ketone body oxidation might attribute to a potential protective mechanism for ensuring the preferential supply of ketone bodies to the brain. Other alternative energy sources such as amino acids and acetyl-CoAs are available for other organs to sustain energy production and biomolecules synthesis. Here, we could not confirm this speculation as we did not assess the ketone body utilization in the brain of cachexia mice. Nevertheless, our study could be an innovative supplementation for clarifying the molecular mechanisms of skeletal muscle atrophy in cancer cachexia. Expectedly, it is of great value to exploit ketone body metabolism-related enzymes as novel targets for improving ketone body utilization and thereby ameliorating cachectic muscle wasting. It seems that enhancing ketone body utilization rather than simply serving cachectic mice with ketogenetic diets [56], might be a more efficient way to ameliorate muscle wasting in cancer cachexia.

\section{Conclusions}

We have demonstrated that amiloride is a potential drug capable of ameliorating muscle wasting in cancer cachexia. Our results reveal that the amiloride treatment significantly improves metabolic impairments in cachectic gastrocnemius through efficiently inhibiting tumorderived exosome release, including promoted muscular catabolism, inhibited muscular protein synthesis, blocked glycolysis, and impeded ketone body oxidation. Our results are beneficial to mechanistic understanding the effects of the amiloride treatment for ameliorating muscle wasting in cancer cachexia and alleviating the CAC progression. Our study sheds light on the potentials of amiloride in cachexia therapy. Further studies are needed both to validate the practical universalities of the amiloride treatment for other cancer cachexia models, and to explore clinical potentials of amiloride for improving the CAC treatment.

\section{Abbreviations}

CAC: Cancer cachexia; NOR: Normal control; AM: Amiloride treated; KD: Rab27 knock-down; MVBs: Multi-vesicular bodies; CM: Conditioned media; HSFCM: High-sensitivity nano flow cytometer; FID: Free induction delay; DEGs: Differentially expressed genes; FC: Fold change; FDR: False discovery rate; TFBWs: Tumor-free body weights; HLMWs: Hind limb muscle weights; PCA: Principal components analysis; PLS-DA: Supervised partial leastsquares discriminant analysis; OXCT1: 3-Oxoacid CoA transferase 1; $\mathrm{BDH} 2$ : 3Hydroxybutyrate dehydrogenase 2; FFA: Free fatty acid; FAA: Free amino 
acid; KB: Ketone body; a-KG: a-Ketoglutarate; AcAc: Acetoacetic acid; F-1,6BP: Fructose-1,6-bisphosphate; G3P: Glyceraldehyde-3-phosphate; DHAP: Dihydroxyacetone phosphate; 3PG: 3-Phosphoglycerate; 2PG: 2Phosphoglycerate; PEP: Phosphoenolpyruvate; 3-HB: 3-Hydroxybutyrate; IMP: Inosine monophosphate

\section{Supplementary Information}

The online version contains supplementary material available at https://doi. org/10.1186/s13395-021-00274-5.

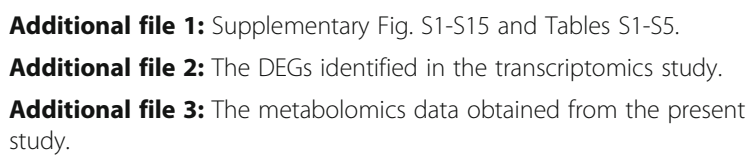

\section{Acknowledgements}

We thank Prof. Xiaomei Yan from College of Chemistry and Chemical Engineering, Xiamen University, for kindly providing the apparatus of HSFCM.

\section{Authors' contributions}

L.Z., D.L., and C.H. conceived this project. L.Z., T.Z., R.L., S.L., H.Z., and H.L. performed the experiments. H.Z. and W.L. provided clinical samples. L.Z., C.H., W.S. B.J, Q.L., and D.L. performed the data analyses and helped with the discussions. L.Z., D.L., and C.H. wrote this manuscript. All authors commented on the manuscript. The authors read and approved the final manuscript.

\section{Funding}

This work was supported by the National Natural Science Foundation of China (No. 31971357) and the Open Research Fund of State Key Laboratory of Cellular Stress Biology, Xiamen University (SKLCSB2020KF002)

\section{Availability of data and materials}

Transcriptome datasets can be found with a GEO accession number: GSE173250. Other datasets supporting the conclusions of this article are included within the article and its additional files.

\section{Declarations}

The authors declare that the research was conducted in the absence of any commercial or financial relationships that could be construed as a potentia conflict of interest.

\section{Ethics approval and consent to participate}

All animal studies were performed in Xiamen University Laboratory Animal Center, according to protocols approved by the Institutional Animal Care and Use Committee of Xiamen University. The study protocol of collecting blood samples of cancer patients was approved by the Ethics Committee of Affiliated Zhongshan Hospital of Xiamen University, China. Patients with colorectal cancer, lung cancer, and gastric cancer were enrolled, which were informed and wrote the consent.

\section{Consent for publication}

Not applicable

\section{Competing interests}

The authors declare that they have no competing interests.

\section{Author details}

${ }^{1}$ Key Laboratory for Chemical Biology of Fujian Province, MOE Key Laboratory of Spectrochemical Analysis \& Instrumentation, College of Chemistry and Chemical Engineering, Xiamen University, Xiamen 361005, China. ${ }^{2}$ Xiamen Cardiovascular Hospital, Xiamen University, Xiamen 361000, China.

${ }^{3}$ Department of Oncology, Institute of Gastrointestinal Oncology, Zhongshan Hospital, Xiamen University, Xiamen 361004, China. ${ }^{4}$ State Key Laboratory of Cellular Stress Biology, School of Life Sciences, Xiamen University, Xiamen 361102, China. ${ }^{5}$ Department of Medical Oncology, Xiang'an Hospital of Xiamen University, Xiamen, China. ${ }^{6}$ Department of Gastrointestinal Surgery, The Affiliated Zhongshan Hospital, Xiamen University, Xiamen 361004, Fujian, China. ${ }^{7}$ Collaborative Innovation Center of Chemistry for Energy Materials, College of Chemistry and Chemical Engineering, Xiamen University, Xiamen
361005, China. ${ }^{8}$ Research and Communication Center of Exercise and Health, Xiamen University of Technology, Xiamen 361024, China. ${ }^{9}$ High-field NMR Center, College of Chemistry and Chemical Engineering, Xiamen University, Xiamen 361005, China.

Received: 17 January 2021 Accepted: 23 June 2021

Published online: 06 July 2021

\section{References}

1. Fearon K, Strasser F, Anker SD, Bosaeus I, Bruera E, Fainsinger RL, et al. Definition and classification of cancer cachexia: an international consensus. Lancet Oncol. 2011;12(5):489-95. https://doi.org/10.1016/S1470-2045(1 0)70218-7.

2. Porporato PE. Understanding cachexia as a cancer metabolism syndrome. Oncogenesis. 2016;5(2):e200. https://doi.org/10.1038/oncsis.2016.3.

3. Baracos VE, Martin L, Korc M, Guttridge DC, Fearon KCH. Cancer-associated cachexia. Nat Rev Dis Primers. 2018;4(1):17105. https://doi.org/10.1038/ nrdp.2017.105.

4. Lok C. Cachexia: The last illness. Nature. 2015;528(7581):182-3. https://doi. org/10.1038/528182a.

5. World Health Organization. [https://www.who.int/en/news-room/factsheets/detail/cancer]. Accessed 16 Jan 2021.

6. Argilés JM, Busquets S, Stemmler B, López-Soriano FJ. Cancer cachexia: understanding the molecular basis. Nat Rev Cancer. 2014;14(11):754-62. https://doi.org/10.1038/nrc3829.

7. Chitti SV, Fonseka P, Mathivanan S. Emerging role of extracellular vesicles in mediating cancer cachexia. Biochem Soc Trans. 2018;46(5):1129-36. https:// doi.org/10.1042/BST20180213.

8. Sagar G, Sah RP, Javeed N, Dutta SK, Smyrk TC, Lau JS, et al. Pathogenesis of pancreatic cancer exosome-induced lipolysis in adipose tissue. Gut. 2016; 65(7):1165-74. https://doi.org/10.1136/gutjnl-2014-308350.

9. Zhang G, Liu Z, Ding H, Zhou Y, Doan HA, Sin KWT, et al. Tumor induces muscle wasting in mice through releasing extracellular Hsp70 and Hsp90. Nat Commun. 2017:8(1):589. https://doi.org/10.1038/s41467-017-00726-X.

10. Wu Q, Sun S, Li Z, Yang Q, Li B, Zhu S, et al. Tumour-originated exosomal miR-155 triggers cancer-associated cachexia to promote tumour progression. Mol Cancer. 2018;17(1):155. https://doi.org/10.1186/s12943-0180899-5.

11. Tang CM, Presser F, Morad M. Amiloride selectively blocks the low threshold (T) calcium channel. Science. 1988;240(4849):213-5. https://doi.org/10.1126/ science. 2451291.

12. Chalmin F, Ladoire S, Mignot G, Vincent J, Bruchard M, Remy-Martin JP, et al. Membrane-associated Hsp72 from tumor-derived exosomes mediates STAT3-dependent immunosuppressive function of mouse and human myeloid-derived suppressor cells. J Clin Invest. 2010;120(2):457-71. https:// doi.org/10.1172/JCl40483.

13. Fitzner D, Schnaars M, van Rossum D, Krishnamoorthy G, Dibaj P, Bakhti M, et al. Selective transfer of exosomes from oligodendrocytes to microglia by macropinocytosis. J Cell Sci. 2011;124(3):447-58. https://doi.org/10.1242/jcs. 074088.

14. He WA, Calore F, Londhe P, Canella A, Guttridge DC, Croce CM Microvesicles containing miRNAs promote muscle cell death in cancer cachexia via TLR7. Proc Natl Acad Sci U S A. 2014;111(12):4525-9. https:// doi.org/10.1073/pnas.1402714111.

15. Tian Y, Ma L, Gong M, Su G, Zhu S, Zhang W, et al. Protein profiling and sizing of extracellular vesicles from colorectal cancer patients via flow cytometry. ACS Nano. 2018;12(1):671-80. https://doi.org/10.1021/acsnano. 7 b07782.

16. Dragovic RA, Gardiner C, Brooks AS, Tannetta DS, Ferguson DJ, Hole P, et al Sizing and phenotyping of cellular vesicles using Nanoparticle Tracking Analysis. Nanomedicine. 2011;7(6):780-8. https://doi.org/10.1016/j.nano.2011. 04.003.

17. Rikkert LG, Nieuwland R, Terstappen L, Coumans FAW. Quality of extracellular vesicle images by transmission electron microscopy is operator and protocol dependent. J Extracell Vesicles. 2019;8(1):1555419. https://doi. org/10.1080/20013078.2018.1555419.

18. Cui P, Shao W, Huang C, Wu CJ, Jiang B, Lin D. Metabolic derangements of skeletal muscle from a murine model of glioma cachexia. Skelet Muscle. 2019;9(1):3. https://doi.org/10.1186/s13395-018-0188-4.

19. Cui P, Huang C, Guo J, Wang Q, Liu Z, Zhuo H, et al. Metabolic profiling of tumors, sera, and skeletal muscles from an orthotopic murine model of 
gastric cancer associated-cachexia. J Proteome Res. 2019;18(4):1880-92. https://doi.org/10.1021/acs.jproteome.9b00088.

20. Beckonert $\mathrm{O}$, Keun HC, Ebbels TM, Bundy J, Holmes E, Lindon JC, et al. Metabolic profiling, metabolomic and metabonomic procedures for NMR spectroscopy of urine, plasma, serum and tissue extracts. Nat Protoc. 2007; 2(11):2692-703. https://doi.org/10.1038/nprot.2007.376.

21. Rong S, Wang L, Peng Z, Liao Y, Li D, Yang $X$, et al. The mechanisms and treatments for sarcopenia: could exosomes be a perspective research strategy in the future? J Cachexia Sarcopenia Muscle. 2020;11(2):348-65. https://doi.org/10.1002/jcsm.12536.

22. Schmidt SF, Rohm M, Herzig S, Berriel DM. Cancer cachexia: more than skeletal muscle wasting. Trends Cancer. 2018;4(12):849-60. https://doi.org/1 0.1016/j.trecan.2018.10.001.

23. Oxlund CS, Buhl KB, Jacobsen IA, Hansen MR, Gram J, Henriksen JE, et al. Amiloride lowers blood pressure and attenuates urine plasminogen activation in patients with treatment-resistant hypertension. J Am Soc Hypertens. 2014;8(12):872-81. https://doi.org/10.1016/j.jash.2014.09.019.

24. Fuchs SC, Poli-de-Figueiredo CE, Figueiredo Neto JA, Scala LC, Whelton PK, Mosele F, et al. Effectiveness of chlorthalidone plus amiloride for the prevention of hypertension: the PREVER-prevention randomized clinical trial. J Am Heart Assoc. 2016;5(12). https://doi.org/10.1161/JAHA.116.004248.

25. Fuchs FD, Scala LCN, Vilela-Martin JF, Whelton PK, Poli-de-Figueiredo CE, Pereira ESR, et al. Effectiveness of chlorthalidone/amiloride versus losartan in patients with stage I hypertension and diabetes mellitus: results from the PREVER-treatment randomized controlled trial. Acta Diabetol. 2020.

26. Chang WH, Liu TC, Yang WK, Lee CC, Lin YH, Chen TY, et al. Amiloride modulates alternative splicing in leukemic cells and resensitizes BcrAblT315I mutant cells to imatinib. Cancer Res. 2011;71(2):383-92. https://doi. org/10.1158/0008-5472.CAN-10-1037.

27. Rojas EA, Corchete LA, San-Segundo L, Martínez-Blanch JF, Codoñer FM, Paíno T, et al. Amiloride, an old diuretic drug, is a potential therapeutic agent for multiple myeloma. Clin Cancer Res. 2017;23(21):6602-15. https:// doi.org/10.1158/1078-0432.CCR-17-0678.

28. Hong Y, Lee $\mathrm{JH}$, Jeong KW, Choi CS, Jun HS. Amelioration of muscle wasting by glucagon-like peptide-1 receptor agonist in muscle atrophy. J Cachexia Sarcopenia Muscle. 2019;10(4):903-18. https://doi.org/10.1002/ jcsm.12434.

29. Huang WC, Kuo KT, Bamodu OA, Lin YK, Wang CH, Lee KY, Wang LS, Yeh CT, Tsai JT. Astragalus polysaccharide (PG2) Ameliorates Cancer Symptom Clusters, as well as Improves Quality of Life in Patients with Metastatic Disease, through Modulation of the Inflammatory Cascade. Cancers (Basel). 2019, 11.

30. Chen JA, Splenser A, Guillory B, Luo J, Mendiratta M, Belinova B, et al. Ghrelin prevents tumour- and cisplatin-induced muscle wasting: characterization of multiple mechanisms involved. J Cachexia Sarcopenia Muscle. 2015;6(2):132-43. https://doi.org/10.1002/jcsm.12023.

31. Del Fabbro E, Inui A, Strasser F. Managing cancer cachexia. In: Cancer Cachexia. Tarporley: Springer Healthcare Ltd; 2012. p. 51-72.

32. Busquets S, Serpe R, Sirisi S, Toledo M, Coutinho J, Martínez R, et al. Megestrol acetate: its impact on muscle protein metabolism supports its use in cancer cachexia. Clin Nutr. 2010;29(6):733-7. https://doi.org/10.1016/j. clnu.2010.06.003.

33. Argilés JM, Anguera A, Stemmler B. A new look at an old drug for the treatment of cancer cachexia: megestrol acetate. Clin Nutr. 2013;32(3):31924. https://doi.org/10.1016/j.clnu.2013.01.004.

34. Zhou X, Wang JL, Lu J, Song Y, Kwak KS, Jiao Q, et al. Reversal of cancer cachexia and muscle wasting by ActRIIB antagonism leads to prolonged survival. Cell. 2010;142(4):531-43. https://doi.org/10.1016/j.cell.2010.07.011.

35. Tisdale MJ. Reversing cachexia. Cell. 2010;142(4):511-2. https://doi.org/10.101 6/j.cell.2010.08.004.

36. Chen $X$, Wu $Y$, Yang $T$, Wei $M$, Wang $Y$, Deng $X$, et al. Salidroside alleviates cachexia symptoms in mouse models of cancer cachexia via activating mTOR signalling. J Cachexia Sarcopenia Muscle. 2016;7(2):225-32. https:// doi.org/10.1002/jcsm.12054.

37. Cheung WW, Hao S, Wang Z, Ding W, Zheng R, Gonzalez A, et al. Vitamin D repletion ameliorates adipose tissue browning and muscle wasting in infantile nephropathic cystinosis-associated cachexia. J Cachexia Sarcopenia Muscle. 2020;11(1):120-34. https://doi.org/10.1002/jcsm.12497.

38. Argilés JM, López-Soriano FJ, Stemmler B, Busquets S. Novel targeted therapies for cancer cachexia. Biochem J. 2017:474(16):2663-78. https://doi. org/10.1042/BCJ20170032.
39. Andersen H, Hansen PB, Bistrup C, Nielsen F, Henriksen JE, Jensen BL. Significant natriuretic and antihypertensive action of the epithelial sodium channel blocker amiloride in diabetic patients with and without nephropathy. J Hypertens. 2016;34(8):1621-9. https://doi.org/10.1097/HJH. 0000000000000967

40. Cameron IL, Hunter KE. Effect of cancer cachexia and amiloride treatment on the intracellular sodium content in tissue cells. Cancer Res. 1983;43(3): 1074-8.

41. Kim KM, Lee YJ. Amiloride augments TRAIL-induced apoptotic death by inhibiting phosphorylation of kinases and phosphatases associated with the P13K-Akt pathway. Oncogene. 2005;24(3):355-66. https://doi.org/10.1038/sj. onc.1208213.

42. Harguindey S, Pedraz JL, García Cañero R, Pérez de Diego J, Cragoe EJ, Jr. Hydrogen ion-dependent oncogenesis and parallel new avenues to cancer prevention and treatment using a $\mathrm{H}(+)$-mediated unifying approach: $\mathrm{pH}-$ related and pH-unrelated mechanisms. Crit Rev Oncog. 1995, 6:1-33.

43. Iorio J, Duranti C, Lottini T, Lastraioli E, Bagni G, Becchetti A, et al. K(V)11.1 Potassium channel and the $\mathrm{Na}(+) / \mathrm{H}(+)$ antiporter NHE1 modulate adhesiondependent intracellular pH in colorectal cancer cells. Front Pharmacol. 2020; 11:848.

44. Tang JY, Chang HW, Chang JG. Modulating roles of amiloride in irradiationinduced antiproliferative effects in glioblastoma multiforme cells involving Akt phosphorylation and the alternative splicing of apoptotic genes. DNA Cell Biol. 2013;32(9):504-10. https://doi.org/10.1089/dna.2013.1998.

45. Savina A, Furlán M, Vidal M, Colombo MI. Exosome release is regulated by a calcium-dependent mechanism in K562 cells. J Biol Chem. 2003:278(22): 20083-90. https://doi.org/10.1074/jbc.M301642200.

46. Zhou J, Liu B, Liang C, Li Y, Song YH. Cytokine signaling in skeletal muscle wasting. Trends Endocrinol Metab. 2016;27(5):335-47. https://doi.org/10.101 6/j.tem.2016.03.002.

47. Argilés JM, Stemmler B, López-Soriano FJ, Busquets S. Inter-tissue communication in cancer cachexia. Nature Reviews Endocrinology. 2019; 15(1):9-20. https://doi.org/10.1038/s41574-018-0123-0.

48. Yang J, Zhang Z, Zhang Y, Ni X, Zhang G, Cui X, Liu M, Xu C, Zhang Q, Zhu H, et al. ZIP4 promotes muscle wasting and cachexia in mice with orthotopic pancreatic tumors by stimulating RAB27B-regulated release of extracellular vesicles from cancer cells Gastroenterology. 2019, 156:722-734.e726.

49. Hu W, Ru Z, Xiao W, Xiong Z, Wang C, Yuan C, et al. Adipose tissue browning in cancer-associated cachexia can be attenuated by inhibition of exosome generation. Biochem Biophys Res Commun. 2018;506(1):122-9. https://doi.org/10.1016/j.bbrc.2018.09.139.

50. Trajkovic K, Hsu C, Chiantia S, Rajendran L, Wenzel D, Wieland F, et al. Ceramide triggers budding of exosome vesicles into multivesicular endosomes. Science. 2008:319:1244-7.

51. Luberto C, Hassler DF, Signorelli P, Okamoto $Y$, Sawai H, Boros E, et al. Inhibition of tumor necrosis factor-induced cell death in MCF7 by a novel inhibitor of neutral sphingomyelinase. J Biol Chem. 2002;277(43):41128-39. https://doi.org/10.1074/jbc.M206747200.

52. Feng D, Zhao WL, Ye YY, Bai XC, Liu RQ, Chang LF, et al. Cellular internalization of exosomes occurs through phagocytosis. Traffic. 2010;11(5): 675-87. https://doi.org/10.1111/j.1600-0854.2010.01041.x.

53. Svensson KJ, Christianson HC, Wittrup A, Bourseau-Guilmain E, Lindqvist $E$, Svensson LM, et al. Exosome uptake depends on ERK1/2-heat shock protein 27 signaling and lipid Raft-mediated endocytosis negatively regulated by caveolin-1. J Biol Chem. 2013;288(24):17713-24. https://doi.org/10.1074/jbc. M112.445403.

54. Sanvee GM, Panajatovic MV, Bouitbir J, Krähenbühl S. Mechanisms of insulin resistance by simvastatin in C2C12 myotubes and in mouse skeletal muscle. Biochem Pharmacol. 2019;164:23-33. https://doi.org/10.1016/j.bcp.2019.02.02 5.

55. Palus S, von Haehling S, Flach VC, Tschirner A, Doehner W, Anker SD, et al. Simvastatin reduces wasting and improves cardiac function as well as outcome in experimental cancer cachexia. Int J Cardiol. 2013;168(4):3412-8. https://doi.org/10.1016/j.ijcard.2013.04.150.

56. Koutnik AP, Poff AM, Ward NP, DeBlasi JM, Soliven MA, Romero MA, Roberson PA, Fox CD, Roberts MD, D'Agostino DP. Ketone bodies attenuate wasting in models of atrophy. J Cachexia Sarcopenia Muscle. 2020.

\section{Publisher's Note}

Springer Nature remains neutral with regard to jurisdictional claims in published maps and institutional affiliations. 